\title{
Impact of Scalar Leptoquarks on Heavy Baryonic Decays
}

\author{
K. Azizi, ${ }^{1}$ A. T. Olgun, ${ }^{2}$ and Z. Tavukoğlü ${ }^{2}$ \\ ${ }^{1}$ Department of Physics, Doğuş University, Acıbadem-Kadıköy, 34722 İstanbul, Turkey \\ ${ }^{2}$ Vocational School, Okan University, Kadıköy Campus, Hasanpaşa-Kadıköy, 34722 İstanbul, Turkey \\ Correspondence should be addressed to K. Azizi; kazizi@dogus.edu.tr
}

Received 2 June 2017; Revised 1 August 2017; Accepted 29 August 2017; Published 9 October 2017

Academic Editor: Alexey A. Petrov

Copyright (c) $2017 \mathrm{~K}$. Azizi et al. This is an open access article distributed under the Creative Commons Attribution License, which permits unrestricted use, distribution, and reproduction in any medium, provided the original work is properly cited. The publication of this article was funded by SCOAP ${ }^{3}$.

We present a study on the impact of scalar leptoquarks on the semileptonic decays of $\Lambda_{b}, \Sigma_{b}$, and $\Xi_{b}$. To this end, we calculate the differential branching ratio and lepton forward-backward asymmetry defining the processes $\Lambda_{b} \rightarrow \Lambda \ell^{+} \ell^{-}, \Sigma_{b} \rightarrow \Sigma \ell^{+} \ell^{-}$, and $\Xi_{b} \rightarrow \Xi \ell^{+} \ell^{-}$, with $\ell$ being $\mu$ or $\tau$, using the form factors calculated via light cone QCD in full theory. In calculations, the errors of form factors are taken into account. We compare the results obtained in leptoquark model with those of the standard model as well as the existing lattice QCD predictions and experimental data.

\section{Introduction}

The physics of transitions based on $b \rightarrow s \ell^{+} \ell^{-}$at quark level constitute one of the main directions of the research in high energy and particle physics both theoretically and experimentally as new physics effects can contribute to such decay channels. The flavor changing neutral current (FCNC) transitions of $\Lambda_{b} \rightarrow \Lambda \ell^{+} \ell^{-}, \Sigma_{b} \rightarrow \Sigma \ell^{+} \ell^{-}$, and $\Xi_{b} \rightarrow$ $\Xi \ell^{+} \ell^{-}$are among important baryonic decay channels that can be used as sensitive probes to indirectly search for new physics contributions. Particularly, the rare $\Lambda_{b} \rightarrow \Lambda \ell^{+} \ell^{-}$ decay channel has been in the focus of much attention in recent years both theoretically and experimentally. The first measurement on the $\Lambda_{b} \rightarrow \Lambda \mu^{+} \mu^{-}$process has been reported by the CDF Collaboration [1] with 24 signal events and a statistical significance of 5.8 Gaussian standard deviations. Using the $p \bar{p}$ collisions data samples corresponding to $6.8 \mathrm{fb}^{-1}$ and $\sqrt{s}=1.96 \mathrm{TeV}$ collected by the CDF II detector, the differential branching ratio for the $\Lambda_{b} \rightarrow$ $\Lambda \mu^{+} \mu^{-}$decay channel has been measured to be $\operatorname{dBr}\left(\Lambda_{b}^{0} \rightarrow\right.$ $\left.\Lambda \mu^{+} \mu^{-}\right) / d q^{2}=[1.73 \pm 0.42$ (stat) \pm 0.55 (syst) $] \times 10^{-6}[1]$. The differential branching fraction of $\Lambda_{b}^{0} \rightarrow \Lambda \mu^{+} \mu^{-}$decay channel has also been measured as $\operatorname{dBr}\left(\Lambda_{b}^{0} \rightarrow \Lambda \mu^{+} \mu^{-}\right) / d q^{2}=$ $\left(1.18_{-0.08}^{+0.09} \pm 0.03 \pm 0.27\right) \times 10^{-7} \mathrm{GeV}^{2} / c^{4}$ at $15 \mathrm{GeV}^{2} / c^{4} \leq$ $q^{2} \leq 20 \mathrm{GeV}^{2} / c^{4}$ region by the LHCb Collaboration [2]. The
LHCb Collaboration has also measured the lepton forwardbackward asymmetries associated with this transition as $A_{\mathrm{FB}}^{\mu}=-0.05 \pm 0.09$ (stat) \pm 0.03 (syst) at $15 \mathrm{GeV}^{2} / c^{4} \leq q^{2} \leq$ $20 \mathrm{GeV}^{2} / c^{4}$ region [2]. The order of branching ratio in $\Lambda_{b} \rightarrow$ $\Lambda e^{+} e^{-}$and $\Lambda_{b} \rightarrow \Lambda \tau^{+} \tau^{-}$as well as in $\Sigma_{b} \rightarrow \Sigma \ell^{+} \ell^{-}$and $\Xi_{b} \rightarrow \Xi \ell^{+} \ell^{-}$(for all leptons) indicates that these channels are all accessible at LHC (for details see [3-7]). We hope that with the RUN II data at the center of mass energy $13 \mathrm{TeV}$ it will be possible to measure different physical quantities related to these FCNC loop level rare transitions in near future.

The LHC RUN II may provide opportunities to search for various new physics scenarios. One of the important new physics models that has been proposed to overcome the problems of some inconsistencies between the SM predictions and experimental data is the leptoquark (LQ) model. Hereafter, by LQ model we mean a minimal renormalizable scalar leptoquark model which will be explained in some details in next section. As an example for the LHC constraints and prospects for scalar leptoquarks explaining the $\bar{B} \rightarrow D^{(*)} \tau \bar{\nu}$ anomaly see [8]. LQs are hypothetical color triplet bosons that couple to leptons and quarks [9]. LQs carry both baryon (B) and lepton (L) quantum numbers with color and electric charges. The spin number of a leptoquark state can be 0 or 1 , corresponding to a scalar leptoquark or vector leptoquark. If the leptoquarks violate both the baryon and lepton numbers, 
they are generally considered to be heavy particles at the level of $\mathcal{O}\left(10^{15}\right) \mathrm{GeV}$ in order to prevent the proton decay. For more detailed information about leptoquark models and the recent experimental and theoretical progress, see [10-30].

In the light of progress about LQs, we calculate the differential branching ratio and lepton forward-backward asymmetry corresponding to $\Lambda_{b} \rightarrow \Lambda \ell^{+} \ell^{-}, \Sigma_{b} \rightarrow \Sigma \ell^{+} \ell^{-}$, and $\Xi_{b} \rightarrow \Xi \ell^{+} \ell^{-}$processes in a scalar LQ model. In the calculations, we use the form factors as the main inputs calculated from the light cone QCD sum rules in full theory. We also encounter the errors of the form factors to the calculations. We compare the regions swept by the LQ model with those of the SM and search for deviations of the LQ model predictions with those of the SM. We also compare the results with the available lattice predictions and experimental data.

The outline of this article is as follows. In next section, we present the effective Hamiltonian responsible for the transitions under consideration both in the SM and LQ models. In Section 3, we present the transition amplitude and matrix elements defining the above transitions. In Section 4, we calculate the differential decay rate and the lepton forwardbackward asymmetry in the baryonic $\Lambda_{b} \rightarrow \Lambda \ell^{+} \ell^{-}, \Sigma_{b} \rightarrow$ $\Sigma \ell^{+} \ell^{-}$, and $\Xi_{b} \rightarrow \Xi \ell^{+} \ell^{-}$channels and numerically analyze the results obtained. We compare the LQ predictions with those of the SM and existing lattice results and experimental data also in this section.

\section{The Effective Hamiltonian and Wilson Coefficients}

At the quark level the effective Hamiltonian, defining the above-mentioned $b \rightarrow s \ell^{+} \ell^{-}$based transitions, in terms of Wilson coefficients and different operators in SM is generally defined as $[32,33]$

$$
\begin{aligned}
& \mathscr{H}_{\mathrm{SM}}^{\mathrm{eff}}=\frac{G_{F} \alpha_{e m} V_{t b} V_{t s}^{*}}{2 \sqrt{2} \pi}\left[C_{9}^{\mathrm{eff}} \bar{s} \gamma_{\mu}\left(1-\gamma_{5}\right) b \bar{\ell} \gamma^{\mu} \ell\right. \\
& +C_{9}^{\mathrm{eff}} \bar{s} \gamma_{\mu}\left(1+\gamma_{5}\right) b \bar{\ell} \gamma^{\mu} \ell+C_{10} \bar{s} \gamma_{\mu}\left(1-\gamma_{5}\right) b \bar{\ell} \gamma^{\mu} \gamma_{5} \ell \\
& +C_{10}^{\prime} \bar{s} \gamma_{\mu}\left(1+\gamma_{5}\right) b \bar{\ell} \gamma^{\mu} \gamma_{5} \ell \\
& -2 m_{b} C_{7}^{\mathrm{eff}} \frac{1}{q^{2}} \bar{s} i \sigma_{\mu \nu} q^{\nu}\left(1+\gamma_{5}\right) b \bar{\ell} \gamma^{\mu} \ell \\
& \left.-2 m_{b} C_{7}^{\prime \mathrm{eff}} \frac{1}{q^{2}} \bar{s} i \sigma_{\mu \nu} q^{\nu}\left(1-\gamma_{5}\right) b \bar{\ell} \gamma^{\mu} \ell\right]
\end{aligned}
$$

where $G_{F}$ is the Fermi weak coupling constant, $\alpha_{e m}$ is the fine structure constant at $Z$ mass scale, $V_{t b}$ and $V_{t s}^{*}$ are elements of the Cabibbo-Kobayashi-Maskawa (CKM) matrix, $C_{9}^{(\prime) \text { eff }}$, $C_{10}^{(\prime)}$, and $C_{7}^{(\prime) \text { eff }}$ are the SM Wilson coefficients, and $q^{2}$ is the transferred momentum squared. Here the superscript "eff" refers to the shifts in the corresponding coefficients due to the effects of four-quark operators at large $q^{2}$. The primed coefficients are ignored since the Hamiltonian does not receive any contribution from the corresponding operators in the SM. We collect the explicit expressions of the Wilson coefficients $C_{9}^{\text {eff }}, C_{10}$, and $C_{7}^{\text {eff }}$ in Appendix A.

Considering the additional contributions arising from the exchange of scalar leptoquarks, the effective Hamiltonian is modified. The modified Hamiltonian in LQ model is obtained from (1) by the replacements $C_{9}^{\text {eff }} \rightarrow C_{9}^{\text {eff, tot }}, C_{9}^{\prime \text { eff }} \rightarrow$ $C_{9}^{\text {eff,tot }}, C_{10} \rightarrow C_{10}^{\text {tot }}$, and $C_{10}^{\prime} \rightarrow C_{10}^{\text {tot }}$. Here, $C_{9}^{\text {eff,tot }}, C_{9}^{\text {eff,tot }}$, $C_{10}^{\text {tot }}$, and $C_{10}^{\text {tot }}$, with the superscript "tot" being referring to "total," are new Wilson coefficients. These coefficients contain contributions from both the SM and LQ models. Note that the Wilson coefficients $C_{7}^{\text {eff }}$ and $C_{7}^{\prime \text { eff }}$ remain unchanged compared to the SM. The new Wilson coefficients are given as (for details see, for instance, [20, 22-25])

$$
\begin{aligned}
C_{9}^{\text {eff,tot }} & =C_{9}^{\text {eff }}+C_{9}^{\mathrm{LQ}}, \\
C_{9}^{\text {eff,tot }} & =C_{9}^{\prime \text { eff }}+C_{9}^{\mathrm{LQ}}, \\
C_{10}^{\mathrm{tot}} & =C_{10}+C_{10}^{\mathrm{LQ}}, \\
C_{10}^{\text {tot }} & =C_{10}^{\prime}+C_{10}^{\mathrm{LQ}},
\end{aligned}
$$

where the coefficients $C_{9}^{\mathrm{LQ}}$ and $C_{10}^{\mathrm{LQ}}$ receive contributions from the exchange of the scalar leptoquarks $X^{(7 / 6)}=$ $(3,2,7 / 6)$ but the primed Wilson coefficients $C_{9}^{\text {LQ }}$ and $C_{10}^{\prime L Q}$ pick up contributions from the exchange of the scalar leptoquarks $X^{(1 / 6)}=(3,2,1 / 6)$. Here we should remark that we consider the effects of the above two scalar leptoquarks on the Wilson coefficients since this representation does not allow for proton decay at tree-level. We do not consider the effects of the vector leptoquarks on the processes under consideration. Hence, in the present study we consider the minimal renormalizable scalar leptoquark models including one single additional representation of $\mathrm{SU}(3) \times \mathrm{SU}(2) \times$ $U(1)$ which guarantees that the proton does not decay. This requisite can only be satisfied by the models that have the representation of $X^{(7 / 6)}=(3,2,7 / 6)$ and $X^{(1 / 6)}=(3,2,1 / 6)$ scalar leptoquarks under the above gauge group (for details see, for instance, $[18,22])$.

Thus the coefficients $C_{9}^{\mathrm{LQ}}$ and $C_{10}^{\mathrm{LQ}}$ are obtained as $[20,22-$ 25]

$$
C_{9}^{\mathrm{LQ}}=C_{10}^{\mathrm{LQ}}=-\frac{\pi}{2 \sqrt{2} G_{F} \alpha_{e m} V_{t b} V_{t s}^{*}} \frac{\lambda_{e}^{23} \lambda_{e}^{22 *}}{M_{Y}^{2}}
$$

and the primed Wilson coefficients $C_{9}^{\prime \mathrm{LQ}}$ and $C_{10}^{\prime \mathrm{LQ}}$ are found as $[20,22-25]$

$$
C_{9}^{\text {LQ }}=-C_{10}^{\prime \mathrm{LQ}}=\frac{\pi}{2 \sqrt{2} G_{F} \alpha_{e m} V_{t b} V_{t s}^{*}} \frac{\lambda_{s}^{22} \lambda_{b}^{32 *}}{M_{V}^{2}},
$$

where $Y$ and $V$ are the two components of doublet LQ, $X=$ $\left(V_{\alpha}, Y_{\alpha}\right)$, with $M_{Y}$ and $M_{V}$ being representing the masses of the components of the scalar leptoquarks (for details on the LQ interaction Lagrangian and corresponding notations see [34]). It is assumed that each individual leptoquark 
contribution to the branching ratio does not exceed the experimental result. Here

$$
0 \leq\left|\frac{\lambda_{e}^{23} \lambda_{e}^{22 *}}{M_{Y}^{2}}\right|=\left|\frac{\lambda_{s}^{22} \lambda_{b}^{32 *}}{M_{V}^{2}}\right| \leq 5 \times 10^{-9} \mathrm{GeV}^{-2},
$$

obtained via the fitting of the model parameters to the $B_{s} \rightarrow$ $\mu^{+} \mu^{-}$data [34]. In (5) we assumed that the contributions of the two components $Y$ and $V$ are equal.

\section{Transition Amplitude and Matrix Elements}

Generally, the amplitude of the transition responsible for the $\Lambda_{b} \rightarrow \Lambda \ell^{+} \ell^{-}, \Sigma_{b} \rightarrow \Sigma \ell^{+} \ell^{-}$, and $\Xi_{b} \rightarrow \Xi \ell^{+} \ell^{-}$ baryonic decays is provided with sandwiching the effective Hamiltonian between the initial and final baryonic states,

$$
\mathscr{M}^{\mathscr{B}_{\mathrm{Q}} \rightarrow \mathscr{B} \ell^{+} \ell^{-}}=\left\langle\mathscr{B}(p)\left|\mathscr{H}^{\mathrm{eff}}\right| \mathscr{B}_{\mathrm{Q}}(p+q, s)\right\rangle,
$$

where $\mathscr{B}$ represents $\Lambda, \Sigma$, and $\Xi$ baryons and $Q$ corresponds to $b$ quark. To get the transition amplitude, we need to consider the following transition matrix elements parametrized in terms of twelve form factors in full QCD, that is, without any expansion in the heavy quark mass or large hadron energies:

$$
\begin{aligned}
& \left\langle\mathscr{B}(p)\left|\bar{s} \gamma_{\mu}\left(1-\gamma_{5}\right) b\right| \mathscr{B}_{\mathrm{Q}}(p+q, s)\right\rangle=\bar{u}_{\mathscr{B}}(p) \\
& \cdot\left[\gamma_{\mu} f_{1}\left(q^{2}\right)+i \sigma_{\mu \nu} q^{\nu} f_{2}\left(q^{2}\right)+q^{\mu} f_{3}\left(q^{2}\right)\right. \\
& \left.-\gamma_{\mu} \gamma_{5} g_{1}\left(q^{2}\right)-i \sigma_{\mu \nu} \gamma_{5} q^{\nu} g_{2}\left(q^{2}\right)-q^{\mu} \gamma_{5} g_{3}\left(q^{2}\right)\right] \\
& \cdot u_{\mathscr{B}_{\mathrm{Q}}}(p+q, s) \text {, } \\
& \left\langle\mathscr{B}(p)\left|\bar{s} \gamma_{\mu}\left(1+\gamma_{5}\right) b\right| \mathscr{B}_{\mathrm{Q}}(p+q, s)\right\rangle=\bar{u}_{\mathscr{B}}(p) \\
& \cdot\left[\gamma_{\mu} f_{1}\left(q^{2}\right)+i \sigma_{\mu \nu} q^{\nu} f_{2}\left(q^{2}\right)+q^{\mu} f_{3}\left(q^{2}\right)\right. \\
& \left.+\gamma_{\mu} \gamma_{5} g_{1}\left(q^{2}\right)+i \sigma_{\mu \nu} \gamma_{5} q^{\nu} g_{2}\left(q^{2}\right)+q^{\mu} \gamma_{5} g_{3}\left(q^{2}\right)\right] \\
& \cdot u_{\mathscr{B}_{\mathrm{Q}}}(p+q, s) \text {, } \\
& \left\langle\mathscr{B}(p)\left|\bar{s} i \sigma_{\mu \nu} q^{\nu}\left(1+\gamma_{5}\right) b\right| \mathscr{B}_{\mathrm{Q}}(p+q, s)\right\rangle=\bar{u}_{\mathscr{B}}(p) \\
& \cdot\left[\gamma_{\mu} f_{1}^{T}\left(q^{2}\right)+i \sigma_{\mu \nu} q^{\nu} f_{2}^{T}\left(q^{2}\right)+q^{\mu} f_{3}^{T}\left(q^{2}\right)\right. \\
& \left.+\gamma_{\mu} \gamma_{5} g_{1}^{T}\left(q^{2}\right)+i \sigma_{\mu \nu} \gamma_{5} q^{\nu} g_{2}^{T}\left(q^{2}\right)+q^{\mu} \gamma_{5} g_{3}^{T}\left(q^{2}\right)\right] \\
& \cdot u_{\mathscr{B}_{\mathrm{Q}}}(p+q, s) \text {, } \\
& \left\langle\mathscr{B}(p)\left|\bar{s} i \sigma_{\mu \nu} q^{\nu}\left(1-\gamma_{5}\right) b\right| \mathscr{B}_{\mathrm{Q}}(p+q, s)\right\rangle=\bar{u}_{\mathscr{B}}(p) \\
& \cdot\left[\gamma_{\mu} f_{1}^{T}\left(q^{2}\right)+i \sigma_{\mu \nu} q^{\nu} f_{2}^{T}\left(q^{2}\right)+q^{\mu} f_{3}^{T}\left(q^{2}\right)\right. \\
& \left.-\gamma_{\mu} \gamma_{5} g_{1}^{T}\left(q^{2}\right)-i \sigma_{\mu \nu} \gamma_{5} q^{\nu} g_{2}^{T}\left(q^{2}\right)-q^{\mu} \gamma_{5} g_{3}^{T}\left(q^{2}\right)\right] \\
& \cdot u_{\mathscr{B}_{\mathrm{Q}}}(p+q, s) \text {, }
\end{aligned}
$$

where $u_{\mathscr{B}_{Q}}$ and $u_{\mathscr{B}}$ represent spinors of the initial and final states, respectively. $f_{i}^{(T)}$ and $g_{i}^{(T)}$ ( $i$ running from 1 to 3 ) are transition form factors. The values of these form factors corresponding to $\Lambda_{b} \rightarrow \Lambda \ell^{+} \ell^{-}, \Sigma_{b} \rightarrow \Sigma \ell^{+} \ell^{-}$, and $\Xi_{b} \rightarrow$ $\Xi \ell^{+} \ell^{-}$transitions and calculated via light cone sum rules in full theory are taken from [3,4] and [5], respectively (for form factors of $\Lambda_{b}$ channel calculated with different phenomenological models see also, for instance, [35-37]). These form factors are also available in lattice QCD in $\Lambda$ channel [31].

Using the above transition matrix elements in terms of form factors, we get the amplitude of the transitions $\Lambda_{b} \rightarrow$ $\Lambda \ell^{+} \ell^{-}, \Sigma_{b} \rightarrow \Sigma \ell^{+} \ell^{-}$, and $\Xi_{b} \rightarrow \Xi \ell^{+} \ell^{-}$in the SM and LQ as

$$
\begin{aligned}
& \mathscr{M}_{\mathrm{SM}}^{\mathscr{B}_{\mathrm{Q}} \rightarrow \mathscr{B} \ell^{+} \ell^{-}}=\frac{G_{F} \alpha_{e m} V_{t b} V_{t s}^{*}}{2 \sqrt{2} \pi}\left\{\left[\bar{u}_{\mathscr{B}}(p)\right.\right. \\
& \cdot\left(\gamma_{\mu}\left[\mathscr{A}_{1}^{\mathrm{SM}} R+\mathscr{B}_{1}^{\mathrm{SM}} L\right]+i \sigma_{\mu \nu} q^{\nu}\left[\mathscr{A}_{2}^{\mathrm{SM}} R+\mathscr{B}_{2}^{\mathrm{SM}} L\right]\right. \\
& \left.\left.+q^{\mu}\left[\mathscr{A}_{3}^{\mathrm{SM}} R+\mathscr{B}_{3}^{\mathrm{SM}} L\right]\right) u_{\mathscr{B}_{\mathrm{Q}}}(p+q, s)\right]\left(\bar{\ell} \gamma^{\mu} \ell\right) \\
& +\left[\overline { u } _ { \mathscr { B } } ( p ) \left(\gamma_{\mu}\left[\mathscr{D}_{1}^{\mathrm{SM}} R+\mathscr{E}_{1}^{\mathrm{SM}} L\right]\right.\right. \\
& \left.+i \sigma_{\mu \nu} q^{\nu}\left[\mathscr{D}_{2}^{\mathrm{SM}} R+\mathscr{E}_{2}^{\mathrm{SM}} L\right]+q^{\mu}\left[\mathscr{D}_{3}^{\mathrm{SM}} R+\mathscr{E}_{3}^{\mathrm{SM}} L\right]\right) \\
& \left.\left.\cdot u_{\mathscr{B}_{\mathrm{Q}}}(p+q, s)\right]\left(\bar{\ell} \gamma^{\mu} \gamma_{5} \ell\right)\right\} \text {, } \\
& \mathscr{M}_{\text {tot }}^{\mathscr{B}_{Q} \rightarrow \mathscr{B} \ell^{+} \ell^{-}}=\frac{G_{F} \alpha_{e m} V_{t b} V_{t s}^{*}}{2 \sqrt{2} \pi}\left\{\left[\bar{u}_{\mathscr{B}}(p)\right.\right. \\
& \cdot\left(\gamma_{\mu}\left[\mathscr{A}_{1}^{\text {tot }} R+\mathscr{B}_{1}^{\text {tot }} L\right]+i \sigma_{\mu \nu} q^{\nu}\left[\mathscr{A}_{2}^{\text {tot }} R+\mathscr{B}_{2}^{\text {tot }} L\right]\right. \\
& \left.\left.+q^{\mu}\left[\mathscr{A}_{3}^{\text {tot }} R+\mathscr{B}_{3}^{\text {tot }} L\right]\right) u_{\mathscr{B}_{\mathrm{Q}}}(p+q, s)\right]\left(\bar{\ell} \gamma^{\mu} \ell\right) \\
& +\left[\overline { u } _ { \mathscr { B } } ( p ) \left(\gamma_{\mu}\left[\mathscr{D}_{1}^{\text {tot }} R+\mathscr{E}_{1}^{\text {tot }} L\right]\right.\right. \\
& \left.+i \sigma_{\mu \nu} q^{\nu}\left[\mathscr{D}_{2}^{\text {tot }} R+\mathscr{E}_{2}^{\text {tot }} L\right]+q^{\mu}\left[\mathscr{D}_{3}^{\text {tot }} R+\mathscr{E}_{3}^{\text {tot }} L\right]\right) \\
& \left.\left.\cdot u_{\mathscr{B}_{\mathrm{Q}}}(p+q, s)\right]\left(\bar{\ell} \gamma^{\mu} \gamma_{5} \ell\right)\right\} \text {, }
\end{aligned}
$$

where $R=\left(1+\gamma_{5}\right) / 2$ and $L=\left(1-\gamma_{5}\right) / 2$ and the calligraphic coefficients are collected in Appendix B.

\section{Physical Observables}

In this section we would like to calculate some physical observables such as the differential decay width, the differential branching ratio, and the lepton forward-backward asymmetry for the considered decay channels.

4.1. The Differential Decay Width. Using the decay amplitudes and transition matrix elements in terms of form factors, we find the differential decay rate defining the transitions under consideration in the LQ model as

$$
\begin{aligned}
& \frac{d^{2} \Gamma_{\text {tot }}}{d \widehat{s} d z}(z, \widehat{s})=\frac{G_{F}^{2} \alpha_{e m}^{2} m_{\mathscr{B}_{Q}}}{16384 \pi^{5}}\left|V_{t b} V_{t s}^{*}\right|^{2} \\
& \cdot v \sqrt{\lambda(1, r, \widehat{s})}\left[\mathscr{T}_{0}^{\text {tot }}(\widehat{s})+\mathscr{T}_{1}^{\text {tot }}(\widehat{s}) z+\mathscr{T}_{2}^{\text {tot }}(\widehat{s}) z^{2}\right],
\end{aligned}
$$


TABLE 1: The values of some input parameters used in our analysis [9].

\begin{tabular}{lc}
\hline Some input parameters & Values \\
\hline$m_{\Lambda_{b}}$ & $5.6195 \mathrm{GeV}$ \\
$m_{\Lambda}$ & $1.11568 \mathrm{GeV}$ \\
$\tau_{\Lambda_{b}}$ & $1.451 \times 10^{-12} \mathrm{~s}$ \\
$m_{\Sigma_{b}}$ & $5.807 \mathrm{GeV}$ \\
$m_{\Sigma}$ & $1.192 \mathrm{GeV}$ \\
$\tau_{\Sigma_{b}}$ & $1.391 \times 10^{-12} \mathrm{~s}$ \\
$m_{\Xi_{b}}$ & $5.791 \mathrm{GeV}$ \\
$m_{\Xi}$ & $1.314 \mathrm{GeV}$ \\
$\tau_{\Xi_{b}}$ & $1.464 \times 10^{-12} \mathrm{~s}$ \\
$m_{W}$ & $80.385 \mathrm{GeV}$ \\
$G_{F}$ & $1.166 \times 10^{-5} \mathrm{GeV}$ \\
$\alpha_{e m}$ & $1 / 137$ \\
$\left|V_{t b} V_{t s}^{*}\right|$ & 0.040 \\
\hline
\end{tabular}

TABLE 2: The values of quark masses in $\overline{M S}$ scheme [9].

\begin{tabular}{lc}
\hline Quarks & Masses in $\overline{M S}$ scheme \\
\hline$m_{c}$ & $(1.275 \pm 0.025) \mathrm{GeV}$ \\
$m_{b}$ & $(4.18 \pm 0.03) \mathrm{GeV}$ \\
$m_{t}$ & $160_{-4.3}^{+4.8} \mathrm{GeV}$ \\
\hline
\end{tabular}

where $v=\sqrt{1-4 m_{\ell}^{2} / q^{2}}$ is the lepton velocity, $\lambda=\lambda(1, r, \widehat{s})=$ $(1-r-\widehat{s})^{2}-4 r \widehat{s}$ is the usual triangle function, $\widehat{s}=q^{2} / m_{\mathscr{B}_{Q}}^{2}, r=$ $m_{\mathscr{B}}^{2} / m_{\mathscr{B}_{Q}}^{2}$, and $z=\cos \theta$ with $\theta$ being the angle between momenta of the lepton $l^{+}$and $\mathscr{B}_{\mathrm{Q}}$ in the center of mass of leptons. The calligraphic $\mathscr{T}_{0}^{\text {tot }}(\widehat{s}), \mathscr{T}_{1}^{\text {tot }}(\widehat{s})$, and $\mathscr{T}_{2}^{\text {tot }}(\widehat{s})$ functions are given in Appendix B.

4.2. The Differential Branching Ratio. Using the expression of the differential decay width, in this subsection, we numerically analyze the differential branching ratio in terms of $q^{2}$ for the decay channels under consideration. For this aim, we present the values of some input parameters and the quark masses in $\overline{M S}$ scheme used in the numerical analysis in Tables 1 and 2 [9]. Using the numerical values in these tables and the expressions presented in Appendix A, we find the values/intervals $C_{7}^{\text {eff }}=-0.295, C_{9}^{\text {eff }}=[1.573,6.625], C_{10}=$ $-4.260, C_{9}^{\text {eff,tot }}=[2.793,4.394], C_{9}^{\text {efff, tot }}=[0,1.586], C_{10}^{\text {tot }}=$ $[-5.846,-4.260]$, and $C_{10}^{\prime \text { tot }}=[-1.586,0]$ for the corresponding Wilson coefficients. Since $C_{9}^{\text {eff(tot) }}$ depend on $q^{2}$, the above intervals for these coefficients denote the maximum and minimum values obtained varying $q^{2}$ in the physical region, that is, $[0-20] \mathrm{GeV}^{2}$. In the case of coefficients with label "tot" the above intervals are obtained considering the intervals for related parameters in (5). Note that we will use directly the expressions of the Wilson coefficients in the numerical analyses instead of the above-mentioned values/intervals. We shall remark that the above-mentioned values/intervals for $C_{7}^{\text {eff }}, C_{9}^{\text {eff }}$, and $C_{10}$ are consistent with the ones obtained in [38-43] for Wilson coefficients using the global fits to $b \rightarrow$

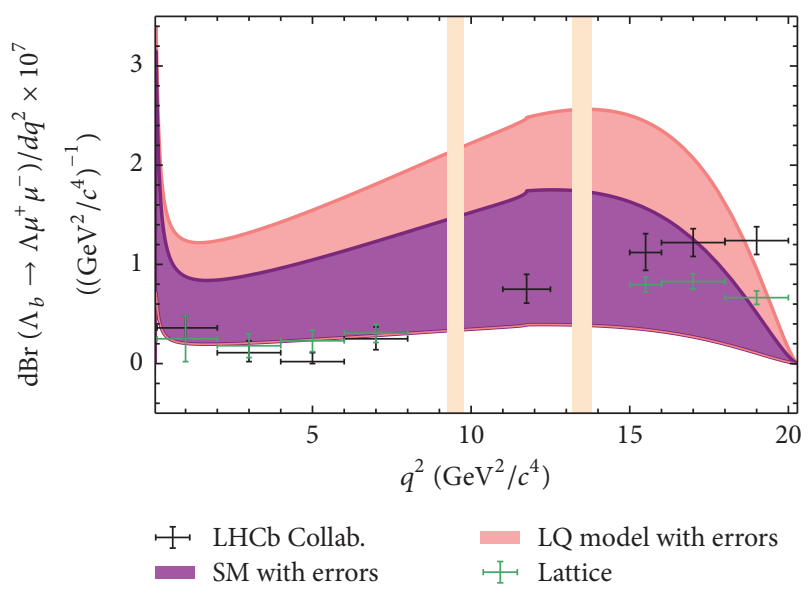

FIGURE 1: The dependence of the differential branching ratio on $q^{2}$ for the $\Lambda_{b} \rightarrow \Lambda \mu^{+} \mu^{-}$transition in the SM and LQ models. The experimental data are taken from the LHCb Collaboration [2]. The lattice predictions are borrowed from [31]. The vertical shaded bands indicate the charmonia veto regions.

$s \ell^{+} \ell^{-}$data. We would also like to compare the intervals for four Wilson coefficients $C_{9}^{\text {eff, tot }}, C_{9}^{\text {'eff, tot }}, C_{10}^{\text {tot }}$, and $C_{10}^{\text {tot }}$, which are relevant to the LQ model with the values extracted in [44] from experimental data on observables of $\Lambda_{b} \rightarrow \Lambda \mu^{+} \mu^{-}$in a $\left(9,10,9^{\prime}, 10^{\prime}\right)$ scenario assuming uncorrelated independent contributions to these coefficients. In [44] the values $C_{9}=$ $6.0_{-0.8}^{+0.8}, C_{9}^{\prime}=0.5_{-1.8}^{+1.3}, C_{10}=-1.3_{-1.1}^{+1.3}$, and $C_{10}^{\prime}=2.3_{-1.3}^{+0.8}$ are obtained. The comparison of the intervals obtained in the present study with those of [44] shows that our prediction on the range of $C_{9}^{\prime}$ exactly remains inside the interval obtained in [44]. For other coefficients although the values obtained in these works are comparable in some regions, we overall see considerable differences between the predictions of two studies. The difference in $C_{9}$ can be attributed to the fact that in [44] the authors use the data only in the interval $q^{2}=$ [15-20] $\mathrm{GeV}^{2}$ to extract its value.

As we previously said, we use the values of form factors calculated via light cone QCD sum rules in full theory and available for all channels under consideration from [35]. These form factors are also available in lattice QCD in $\Lambda$ channel [31]. The differential branching ratios of decay channels under consideration on $q^{2}$, in the SM and LQ models, at $\mu$ and $\tau$ lepton channels are plotted in Figures 1-6. Note that, in these figures, the form factors are encountered with their uncertainties in both models. The bands in LQ model are due to both the constrained regions of some parameters presented in (5) and errors of form factors. In these figures, we show the charmonia veto regions by the vertical shaded bands. We do not present the results for $e$ channel in the figures, because the predictions of $\mu$ channel are very close to those of the $e$ channel. In Figure 1, we also show the experimental data provided by $\mathrm{LHCb}$ [2] and lattice predictions [31]. From these figures it is clear that

(i) the bands of differential branching ratios in terms of $q^{2}$ obtained in SM for all baryonic processes at both lepton channels remain inside the bands of 


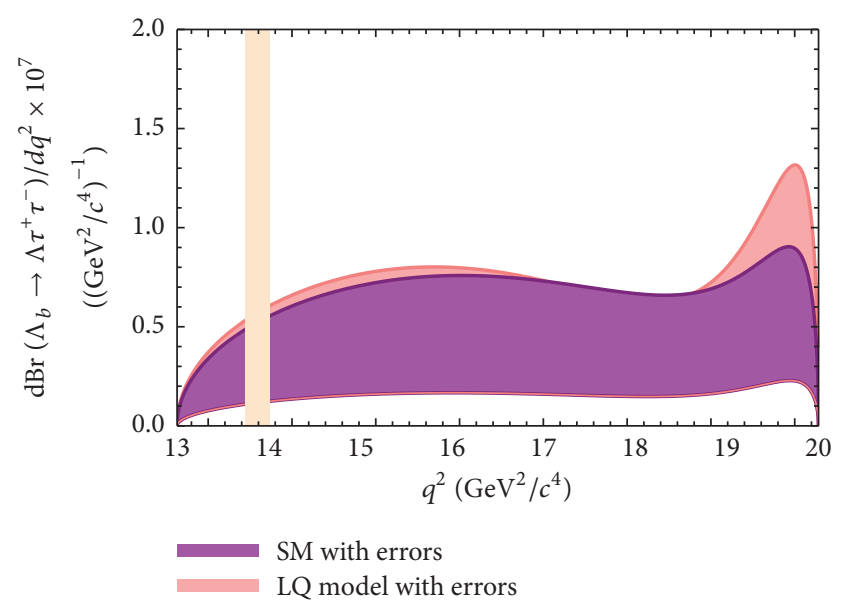

FIgURE 2: The dependence of the differential branching ratio on $q^{2}$ for the $\Lambda_{b} \rightarrow \Lambda \tau^{+} \tau^{-}$transition in the SM and LQ models. The vertical shaded band indicates the charmonia veto region.

the LQ model. The LQ model bands are wider and somewhere show considerable discrepancies from the SM predictions for all channels roughly at whole physical regions of $q^{2}$;

(ii) the SM band for the differential branching fraction in $\Lambda_{b} \rightarrow \Lambda \mu^{+} \mu^{-}$channel roughly coincides with all the lattice predictions borrowed from [31]. This band also defines all the experimental data provided by the LHCb Collaboration except that in the interval $18 \mathrm{GeV}^{2} / c^{4} \leq q^{2} \leq 20 \mathrm{GeV}^{2} / c^{4}$, which can not be described by the SM. This datum coincides with the LQ band. As is also seen from this figure the lattice $\mathrm{QCD}$ predictions on the differential branching fraction in $\Lambda_{b} \rightarrow \Lambda \mu^{+} \mu^{-}$channel show considerable discrepancies with the experimental data in the interval $15 \mathrm{GeV}^{2} / c^{4} \leq q^{2} \leq 20 \mathrm{GeV}^{2} / c^{4}$.

4.3. The Lepton Forward-Backward Asymmetry. In this subsection, we present the results of the lepton forwardbackward asymmetry $\left(\mathscr{A}_{\mathrm{FB}}\right)$ which is one of useful observables to search for NP effects. This quantity is defined as

$$
\begin{aligned}
& \mathscr{A}_{\mathrm{FB}}(\widehat{s}) \\
& =\frac{\int_{0}^{1}\left(d^{2} \Gamma / d \widehat{s} d z\right)(z, \widehat{s}) d z-\int_{-1}^{0}\left(d^{2} \Gamma / d \widehat{s} d z\right)(z, \widehat{s}) d z}{\int_{0}^{1}\left(d^{2} \Gamma / d \widehat{s} d z\right)(z, \widehat{s}) d z+\int_{-1}^{0}\left(d^{2} \Gamma / d \widehat{s} d z\right)(z, \widehat{s}) d z} .
\end{aligned}
$$

In order to see how predictions of LQ scenario deviate from those of the SM, we plot the dependence of the lepton forward-backward asymmetry on $q^{2}$ for the channels under discussion in Figures 7-12. In Figure 7, we also present the measured values of the leptonic forward-backwardasymmetries by the LHCb Collaboration [2] as well as the lattice QCD predictions [31] in the $\Lambda_{b} \rightarrow \Lambda \mu^{+} \mu^{-}$decay channel. From these figures, we read that

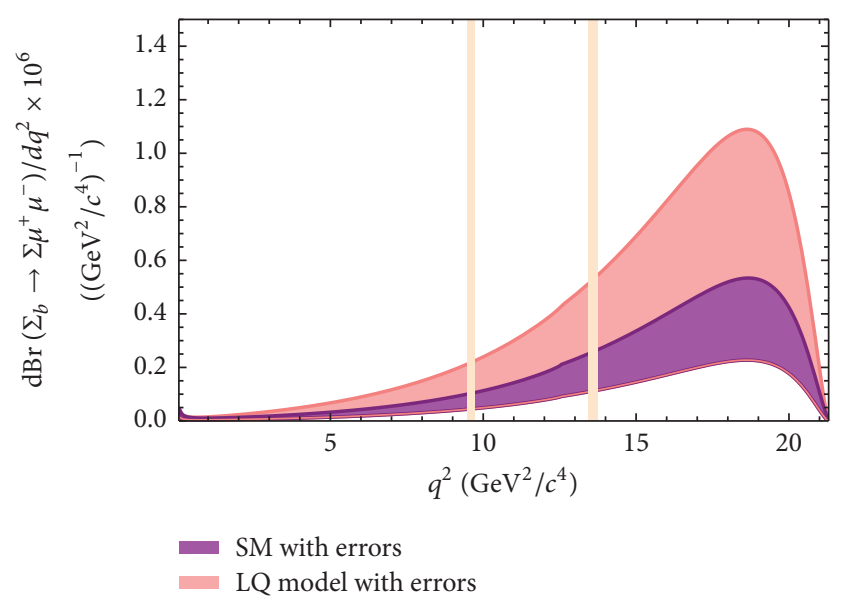

FIGURE 3: The dependence of the differential branching ratio on $q^{2}$ for the $\Sigma_{b} \rightarrow \Sigma \mu^{+} \mu^{-}$transition in the SM and LQ models. The vertical shaded bands indicate the charmonia veto regions.

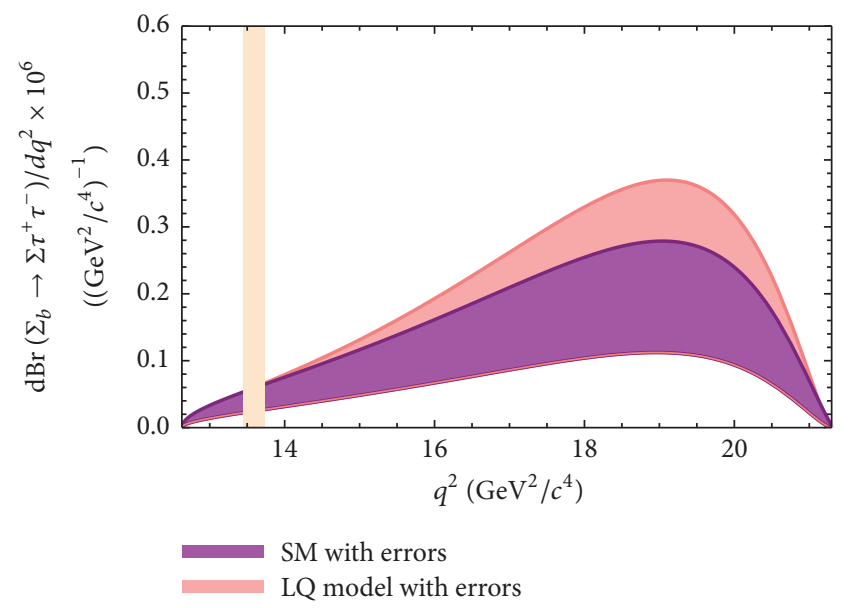

FIGURE 4: The dependence of the differential branching ratio on $q^{2}$ for the $\Sigma_{b} \rightarrow \Sigma \tau^{+} \tau^{-}$transition in the SM and LQ models. The vertical shaded band indicates the charmonia veto region.

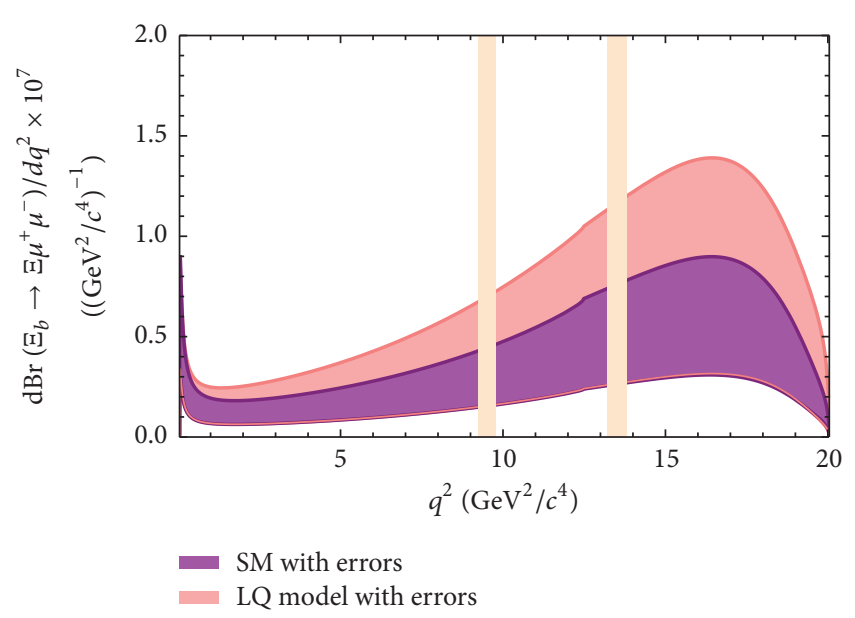

FIgURE 5: The dependence of the differential branching ratio on $q^{2}$ for the $\Xi_{b} \rightarrow \Xi \mu^{+} \mu^{-}$transition in the SM and LQ models. The vertical shaded bands indicate the charmonia veto regions. 


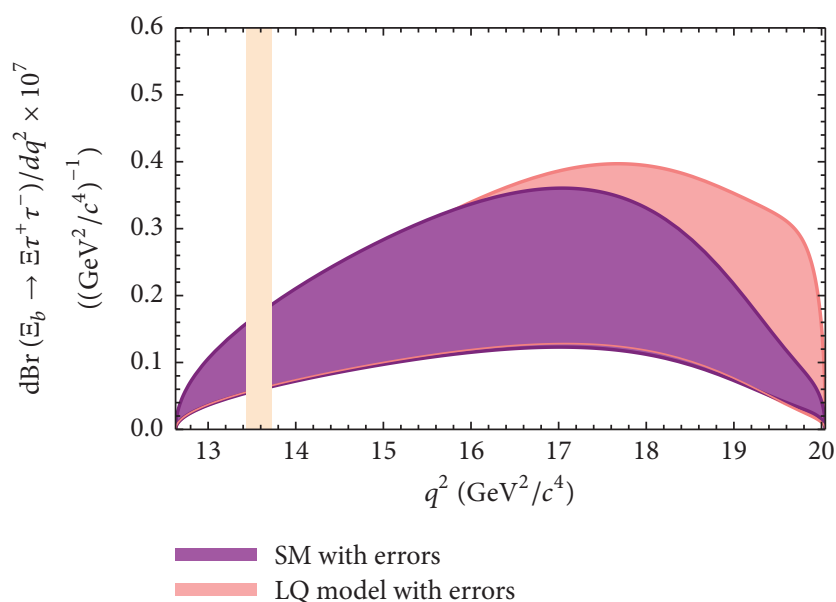

FIGURE 6: The dependence of the differential branching ratio on $q^{2}$ for the $\Xi_{b} \rightarrow \Xi \tau^{+} \tau^{-}$transition in the SM and LQ models. The vertical shaded band indicates the charmonia veto region.

(i) in all decay channels the LQ model predictions demonstrate considerable discrepancies from the SM predictions;

(ii) the SM band on the lepton forward-backward asymmetry in $\Lambda_{b} \rightarrow \Lambda \mu^{+} \mu^{-}$channel coincides with the existing lattice QCD predictions borrowed from [31];

(iii) ignoring from the small intersection of the SM narrow bands with errors of the experimental data at very low and high values of $q^{2}$, the LQ model, against the SM, can describe all data available in $\Lambda_{b} \rightarrow \Lambda \mu^{+} \mu^{-}$ channel. The lattice QCD predictions in this channel also show sizable differences with the experimental data.

\section{Conclusion}

In the present work, we have performed a comprehensive analysis of the semileptonic $\Lambda_{b} \rightarrow \Lambda \ell^{+} \ell^{-}, \Sigma_{b} \rightarrow \Sigma \ell^{+} \ell^{-}$, and $\Xi_{b} \rightarrow \Xi \ell^{+} \ell^{-}$rare processes in the SM as well as the scalar leptoquark model. Using the parametrization of the matrix elements in terms of form factors calculated via light cone QCD sum rules in the full theory, we calculated the differential decay width and numerically analyzed the differential branching fraction and the lepton forward-backward asymmetry in terms of $q^{2}$ in different heavy baryonic decay channels for both $\mu$ and $\tau$ leptons in both scenarios. We compared the predictions of the LQ model on the considered physical observables with those of the SM and the existing lattice QCD predictions as well as experimental data in $\Lambda_{b} \rightarrow$ $\Lambda \mu^{+} \mu^{-}$channel. We observed that the predictions of the LQ model in all channels show considerable discrepancies with those of the SM on both the differential decay width and lepton forward-backward asymmetry. The SM results for both the observables considered in the present study are consistent with the existing predictions of lattice QCD. Except the interval $18 \mathrm{GeV}^{2} / c^{4} \leq q^{2} \leq 20 \mathrm{GeV}^{2} / c^{4}$, the SM band describes the existing experimental data on the

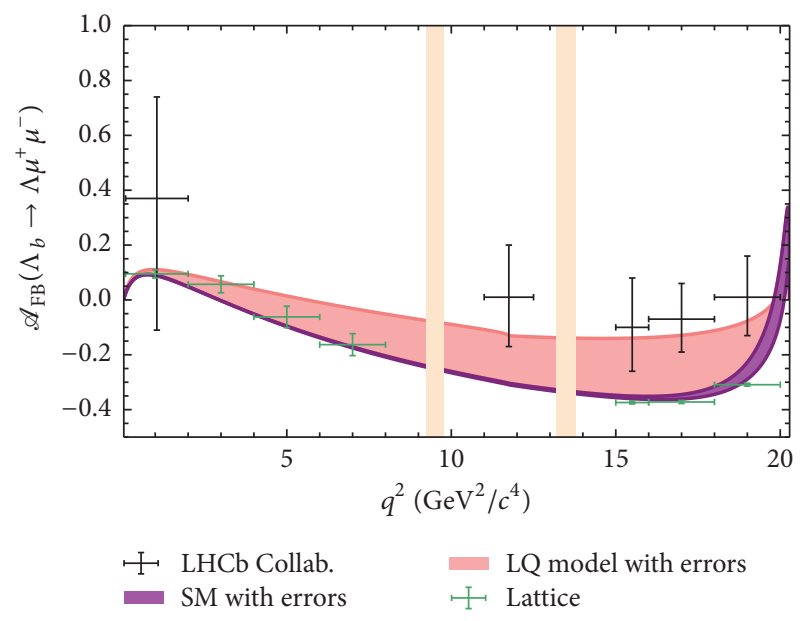

Figure 7: The dependence of the $\mathscr{A}_{\mathrm{FB}}$ on $q^{2}$ for the $\Lambda_{b} \rightarrow \Lambda \mu^{+} \mu^{-}$ transition in the SM and LQ models. The experimental data are taken from the LHCb Collaboration [2]. The lattice predictions are borrowed from [31]. The vertical shaded bands indicate the charmonia veto regions.

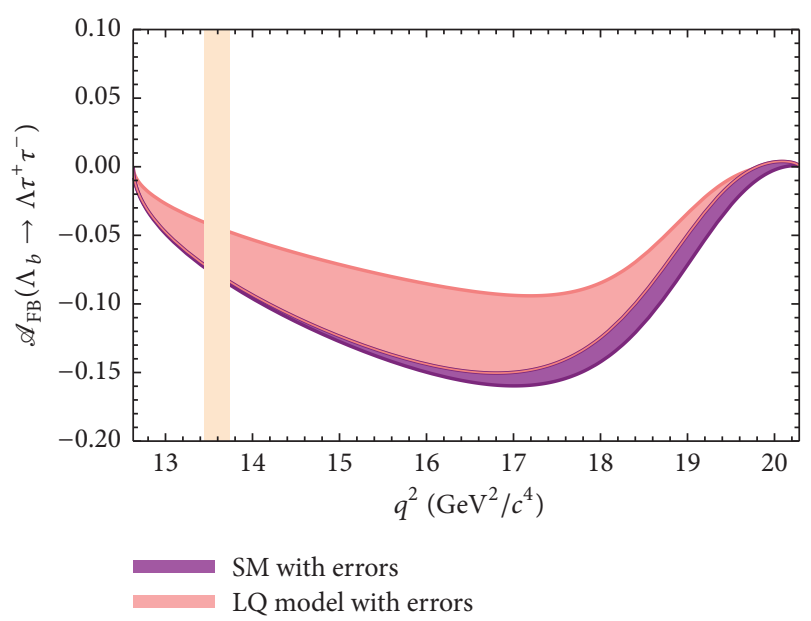

Figure 8: The dependence of $\mathscr{A}_{\mathrm{FB}}$ on $q^{2}$ for the $\Lambda_{b} \rightarrow \Lambda \tau^{+} \tau^{-}$ transition in the SM and LQ models. The vertical shaded band indicates the charmonia veto region.

differential branching ratio in $\Lambda_{b} \rightarrow \Lambda \mu^{+} \mu^{-}$transition. The datum in $18 \mathrm{GeV}^{2} / c^{4} \leq q^{2} \leq 20 \mathrm{GeV}^{2} / c^{4}$ coincides with the LQ model prediction.

In the case of lepton forward-backward asymmetry, the $\mathrm{SM}$, overall, can not describe the experimental data existing in $\Lambda_{b} \rightarrow \Lambda \mu^{+} \mu^{-}$channel, while the LQ model band coincides with the experimental data.

More experimental data in $\Lambda_{b} \rightarrow \Lambda \tau^{+} \tau^{-}$as well as $\Sigma_{b} \rightarrow \Sigma \ell^{+} \ell^{-}$and $\Xi_{b} \rightarrow \Xi \ell^{+} \ell^{-}$with both leptons are needed to compare with the theoretical predictions. We hope that, with the RUN II data, it will be possible to measure different physical quantities related to such FCNC transitions at $\mathrm{LHCb}$ in near future. Comparison of the future experimental data with the theoretical predictions on different physical quantities in various decay channels can help us 


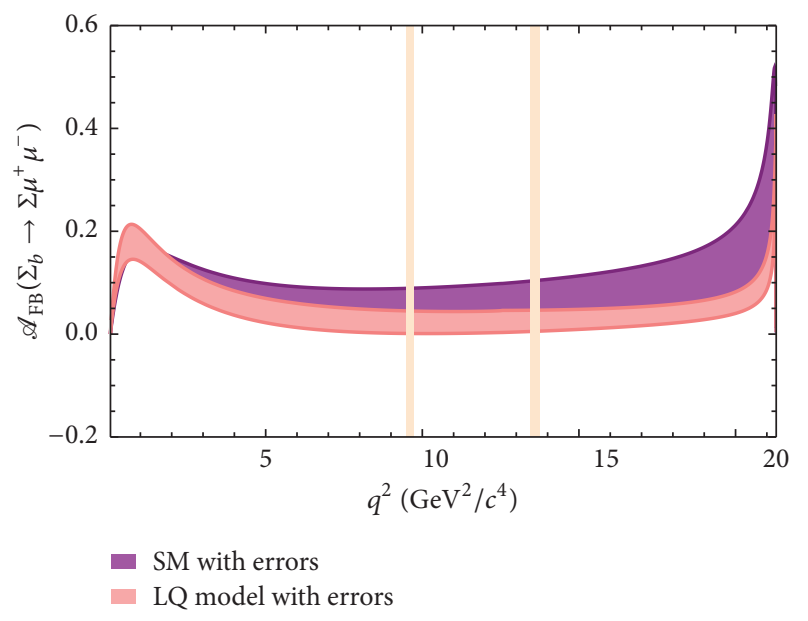

Figure 9: The dependence of $\mathscr{A}_{\mathrm{FB}}$ on $q^{2}$ for the $\Sigma_{b} \rightarrow \Sigma \mu^{+} \mu^{-}$ transition in the SM and LQ models. The vertical shaded bands indicate the charmonia veto regions.

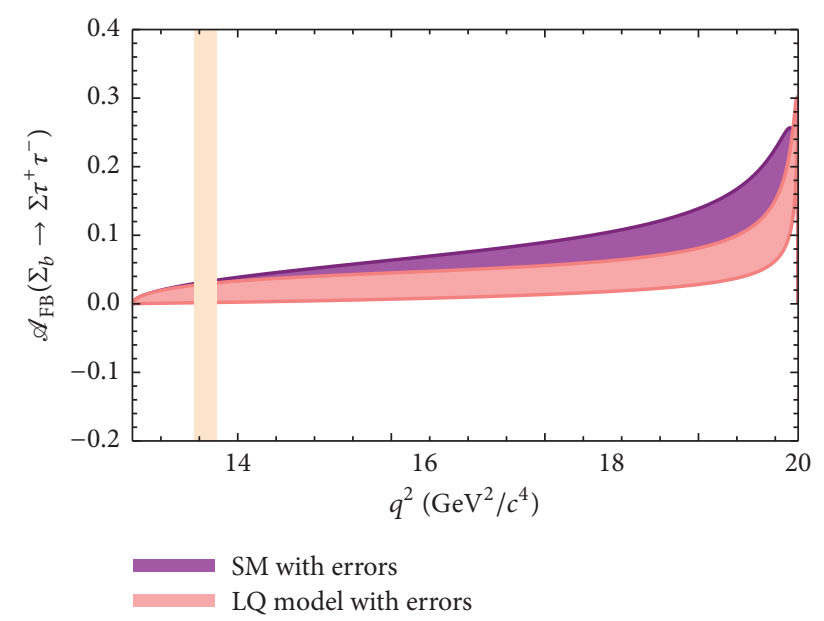

Figure 10: The dependence of $\mathscr{A}_{\mathrm{FB}}$ on $q^{2}$ for the $\Sigma_{b} \rightarrow \Sigma \tau^{+} \tau^{-}$ transition in the SM and LQ models. The vertical shaded band indicates the charmonia veto region.

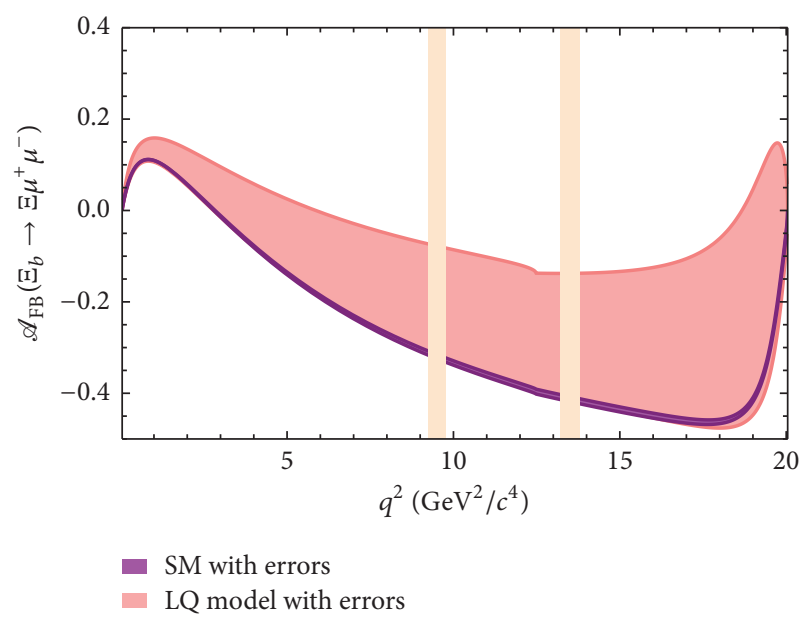

Figure 11: The dependence of $\mathscr{A}_{\mathrm{FB}}$ on $q^{2}$ for the $\Xi_{b} \rightarrow \Xi \mu^{+} \mu^{-}$ transition in the SM and LQ models. The vertical shaded bands indicate the charmonia veto regions.

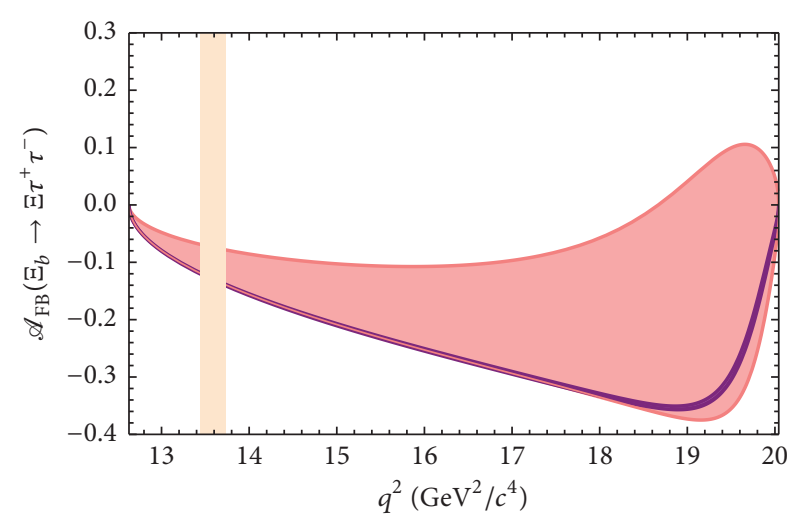

SM with errors

LQ model with errors

FIgURE 12: The dependence of $\mathscr{A}_{\mathrm{FB}}$ on $q^{2}$ for the $\Xi_{b} \rightarrow \Xi \tau^{+} \tau^{-}$ transition in the SM and LQ models. The vertical shaded band indicates the charmonia veto region.

better explain some anomalies between the SM predictions and the experimental data. Any sizable discrepancy between the theoretical predictions on physical observables with the experimental data can be considered as an indication of new physics effects and may help us in the course of searching for the new particles like leptoquarks.

Note Added. When preparing this work we noticed that a part of our work, namely, the $\Lambda_{b} \rightarrow \Lambda \ell^{+} \ell^{-}$channel has been investigated in $[34,45]$ within the same framework. In these studies the authors use the form factors, as the main inputs, calculated in heavy quark effective theory while we use the form factors calculated via light cone QCD sum rules in full theory.

\section{Appendix}

\section{A. The Wilson Coefficients}

The Wilson coefficient $C_{7}^{\text {eff }}$ in leading logarithm approximation in the SM is written by [46-49]

$$
\begin{aligned}
C_{7}^{\mathrm{eff}}\left(\mu_{b}\right)= & \eta^{16 / 23} C_{7}\left(\mu_{W}\right) \\
& +\frac{8}{3}\left(\eta^{14 / 23}-\eta^{16 / 23}\right) C_{8}\left(\mu_{W}\right) \\
& +C_{2}\left(\mu_{W}\right) \sum_{i=1}^{8} h_{i} \eta^{a_{i}},
\end{aligned}
$$

where

$$
\begin{aligned}
& C_{7}\left(\mu_{W}\right)=-\frac{1}{2} D_{0}^{\prime}\left(x_{t}\right), \\
& C_{8}\left(\mu_{W}\right)=-\frac{1}{2} E_{0}^{\prime}\left(x_{t}\right),
\end{aligned}
$$




$$
C_{2}\left(\mu_{W}\right)=1
$$

The functions $D_{0}^{\prime}\left(x_{t}\right)$ and $E_{0}^{\prime}\left(x_{t}\right)$ with $x_{t}=m_{t}^{2} / m_{W}^{2}$ are given as

$$
\begin{aligned}
& D_{0}^{\prime}\left(x_{t}\right)=-\frac{\left(8 x_{t}^{3}+5 x_{t}^{2}-7 x_{t}\right)}{12\left(1-x_{t}\right)^{3}}+\frac{x_{t}^{2}\left(2-3 x_{t}\right)}{2\left(1-x_{t}\right)^{4}} \ln x_{t}, \\
& E_{0}^{\prime}\left(x_{t}\right)=-\frac{x_{t}\left(x_{t}^{2}-5 x_{t}-2\right)}{4\left(1-x_{t}\right)^{3}}+\frac{3 x_{t}^{2}}{2\left(1-x_{t}\right)^{4}} \ln x_{t} .
\end{aligned}
$$

The parameter $\eta$ in (A.1) is defined as

$$
\eta=\frac{\alpha_{s}\left(\mu_{W}\right)}{\alpha_{s}\left(\mu_{b}\right)}
$$

with

$$
\alpha_{s}(x)=\frac{\alpha_{s}\left(m_{Z}\right)}{1-\beta_{0}\left(\alpha_{s}\left(m_{Z}\right) / 2 \pi\right) \ln \left(m_{Z} / x\right)},
$$

where $\alpha_{s}\left(m_{Z}\right)=0.118$ and $\beta_{0}=23 / 3$. The coefficients $h_{i}$ and $a_{i}$ in (A.1) are also written by $[47,48]$

$$
\begin{aligned}
& h_{i}=\left(2.2996,-1.0880,-\frac{3}{7},-\frac{1}{14},-0.6494,-0.0380,-0.0186,-0.0057\right), \\
& a_{i}=\left(\frac{14}{23}, \frac{16}{23}, \frac{6}{23},-\frac{12}{23}, 0.4086,-0.4230,-0.8994,0.1456\right) .
\end{aligned}
$$

The Wilson coefficient $C_{9}^{\text {eff }}$ in $\mathrm{SM}$ is given by $[47,48]$

$$
\begin{aligned}
C_{9}^{\mathrm{eff}}\left(\hat{s}^{\prime}\right) & \\
= & C_{9}^{\mathrm{NDR}} \eta\left(\widehat{s}^{\prime}\right) \\
& +h\left(z, \hat{s}^{\prime}\right)\left(3 C_{1}+C_{2}+3 C_{3}+C_{4}+3 C_{5}+C_{6}\right) \\
& -\frac{1}{2} h\left(1, \hat{s}^{\prime}\right)\left(4 C_{3}+4 C_{4}+3 C_{5}+C_{6}\right) \\
& -\frac{1}{2} h\left(0, \hat{s}^{\prime}\right)\left(C_{3}+3 C_{4}\right) \\
& +\frac{2}{9}\left(3 C_{3}+C_{4}+3 C_{5}+C_{6}\right)
\end{aligned}
$$

where $\hat{s}^{\prime}=q^{2} / m_{b}^{2}$ with $4 m_{l}^{2} \leq q^{2} \leq\left(m_{\mathscr{B}_{Q}}-m_{\mathscr{B}}\right)^{2} . C_{9}^{\mathrm{NDR}}$ in the naive dimensional regularization (NDR) scheme is written as

$$
C_{9}^{\mathrm{NDR}}=P_{0}^{\mathrm{NDR}}+\frac{Y}{\sin ^{2} \theta_{W}}-4 Z+P_{E} E,
$$

where $P_{0}^{\mathrm{NDR}}=2.60 \pm 0.25, \sin ^{2} \theta_{W}=0.23, Y=0.98$, and $Z=0.679$ [47-49]. The last term in (A.8) is ignored due to the negligible value of $P_{E}$. In (A.7), $\eta\left(\widehat{s}^{\prime}\right)$ is given as

$$
\eta\left(\vec{s}^{\prime}\right)=1+\frac{\alpha_{s}\left(\mu_{b}\right)}{\pi} \omega\left(\vec{s}^{\prime}\right),
$$

with

$$
\begin{aligned}
\omega\left(\vec{s}^{\prime}\right)= & -\frac{2}{9} \pi^{2}-\frac{4}{3} \operatorname{Li}_{2}\left(\widehat{s}^{\prime}\right)-\frac{2}{3} \ln \widehat{s}^{\prime} \ln \left(1-\widehat{s}^{\prime}\right) \\
& -\frac{5+4 \widehat{s}^{\prime}}{3\left(1+2 \widehat{s}^{\prime}\right)} \ln \left(1-\vec{s}^{\prime}\right)
\end{aligned}
$$

$$
\begin{aligned}
& -\frac{2 \widehat{s}^{\prime}\left(1+\widehat{s}^{\prime}\right)\left(1-2 \widehat{s}^{\prime}\right)}{3\left(1-\widehat{s}^{\prime}\right)^{2}\left(1+2 \widehat{s}^{\prime}\right)} \ln \widehat{s}^{\prime} \\
& +\frac{5+9 \widehat{s}^{\prime}-6 \vec{s}^{\prime 2}}{6\left(1-\widehat{s}^{\prime}\right)\left(1+2 \widehat{s}^{\prime}\right)} .
\end{aligned}
$$

The function $h\left(y, \widehat{s}^{\prime}\right)$ is written as

$$
\begin{aligned}
& h\left(y, \widehat{s}^{\prime}\right)=-\frac{8}{9} \ln \frac{m_{b}}{\mu_{b}}-\frac{8}{9} \ln y+\frac{8}{27}+\frac{4}{9} x \\
& -\frac{2}{9}(2+x)|1-x|^{1 / 2} \\
& \quad \begin{cases}\left(\ln \left|\frac{\sqrt{1-x}+1}{\sqrt{1-x}-1}\right|-i \pi\right), & \text { for } x \equiv \frac{4 z^{2}}{\widehat{s}^{\prime}}<1 \\
2 \arctan \frac{1}{\sqrt{x-1}}, & \text { for } x \equiv \frac{4 z^{2}}{\widehat{s}^{\prime}}>1,\end{cases}
\end{aligned}
$$

where $y=1$ or $y=z=m_{c} / m_{b}$ and

$$
h\left(0, \hat{s}^{\prime}\right)=\frac{8}{27}-\frac{8}{9} \ln \frac{m_{b}}{\mu_{b}}-\frac{4}{9} \ln \hat{s}^{\prime}+\frac{4}{9} i \pi .
$$

The coefficients $C_{j}(j=1, \ldots, 6)$ at $\mu_{b}=5 \mathrm{GeV}$ scale are also written as [49]

$$
C_{j}=\sum_{i=1}^{8} k_{j i} \eta^{a_{i}} \quad(j=1, \ldots, 6),
$$

where $k_{j i}$ are given as

$$
\begin{aligned}
& k_{1 i}=\left(0,0, \frac{1}{2},-\frac{1}{2}, 0,0,0,0\right), \\
& k_{2 i}=\left(0,0, \frac{1}{2}, \frac{1}{2}, 0,0,0,0\right),
\end{aligned}
$$




$$
\begin{aligned}
& k_{3 i} \\
& =\left(0,0,-\frac{1}{14}, \frac{1}{6}, 0.0510,-0.1403,-0.0113,0.0054\right), \\
& k_{4 i} \\
& =\left(0,0,-\frac{1}{14},-\frac{1}{6}, 0.0984,0.1214,0.0156,0.0026\right), \\
& k_{5 i}=(0,0,0,0,-0.0397,0.0117,-0.0025,0.0304), \\
& k_{6 i}=(0,0,0,0,0.0335,0.0239,-0.0462,-0.0112) .
\end{aligned}
$$

Considering the resonances from $J / \psi$ family, we divide the allowed physical region into the following three regions in the case of the electron and muon as final leptons:

Region I; $4 m_{l}^{2} \leq q^{2} \leq\left(m_{J / \psi(1 s)}-0.02\right)^{2}$

Region II; $\left(m_{J / \psi(1 s)}+0.02\right)^{2} \leq q^{2} \leq\left(m_{\psi(2 s)}-0.02\right)^{2}$,

Region III; $\left(m_{\psi(2 s)}+0.02\right)^{2} \leq q^{2} \leq\left(m_{\mathscr{B}_{Q}}-m_{\mathscr{B}}\right)^{2}$.

In the case of $\tau$, we have the following two regions:

Region I; $4 m_{\tau}^{2} \leq q^{2} \leq\left(m_{\psi(2 s)}-0.02\right)^{2}$

Region II; $\left(m_{\psi(2 s)}+0.02\right)^{2} \leq q^{2} \leq\left(m_{\mathscr{B}_{Q}}-m_{\mathscr{B}}\right)^{2}$.

The Wilson coefficient $C_{10}$ in the $\mathrm{SM}$ is given as

$$
C_{10}=-\frac{Y}{\sin ^{2} \theta_{W}}
$$

\section{B. The Functions Used in Transition Amplitudes and Differential Decay Rate}

The calligraphic coefficients used in the transition amplitudes of the considered processes are find as

$$
\begin{aligned}
\mathscr{A}_{1}= & f_{1} C_{9}^{\mathrm{eff}+}-g_{1} C_{9}^{\mathrm{eff}-} \\
& -2 m_{b} \frac{1}{q^{2}}\left[f_{1}^{T} C_{7}^{\mathrm{eff}+}+g_{1}^{T} C_{7}^{\mathrm{eff}-}\right], \\
\mathscr{A}_{2}= & \mathscr{A}_{1}(1 \longrightarrow 2), \\
\mathscr{A}_{3}= & \mathscr{A}_{1}(1 \longrightarrow 3), \\
\mathscr{B}_{1}= & f_{1} C_{9}^{\mathrm{eff}+}+g_{1} C_{9}^{\mathrm{eff}-} \\
& -2 m_{b} 1 q^{2}\left[f_{1}^{T} C_{7}^{\mathrm{eff}+}-g_{1}^{T} C_{7}^{\mathrm{eff}-}\right],
\end{aligned}
$$

$$
\begin{aligned}
& \mathscr{B}_{2}=\mathscr{B}_{1}(1 \longrightarrow 2), \\
& \mathscr{B}_{3}=\mathscr{B}_{1}(1 \longrightarrow 3), \\
& \mathscr{D}_{1}=f_{1} C_{10}^{+}-g_{1} C_{10}^{-}, \\
& \mathscr{D}_{2}=\mathscr{D}_{1}(1 \longrightarrow 2), \\
& \mathscr{D}_{3}=\mathscr{D}_{1}(1 \longrightarrow 3), \\
& \mathscr{E}_{1}=f_{1} C_{10}^{+}+g_{1} C_{10}^{-}, \\
& \mathscr{E}_{2}=\mathscr{E}_{1}(1 \longrightarrow 2), \\
& \mathscr{E}_{3}=\mathscr{E}_{1}(1 \longrightarrow 3),
\end{aligned}
$$

with

$$
\begin{aligned}
& C_{9}^{\mathrm{eff}+}=C_{9}^{\mathrm{eff}}+C_{9}^{\text {eff }}, \\
& C_{9}^{\mathrm{eff}-}=C_{9}^{\mathrm{eff}}-C_{9}^{\text {eff }}, \\
& C_{7}^{\mathrm{eff}+}=C_{7}^{\mathrm{eff}}+C_{7}^{\text {eff }}, \\
& C_{7}^{\mathrm{eff}-}=C_{7}^{\mathrm{eff}}-C_{7}^{\prime \mathrm{eff}}, \\
& C_{10}^{+}=C_{10}+C_{10}^{\prime}, \\
& C_{10}^{-}=C_{10}-C_{10}^{\prime} .
\end{aligned}
$$

The functions $\mathscr{T}_{0}^{\text {tot }}(\widehat{s}), \mathscr{T}_{1}^{\text {tot }}(\widehat{s})$, and $\mathscr{T}_{2}^{\text {tot }}(\widehat{s})$ in the differential decay width are given as

$$
\begin{aligned}
& \mathscr{T}_{0}^{\text {tot }}(\widehat{s})=32 m_{\ell}^{2} m_{\mathscr{B}_{Q}}^{4} \widehat{s}(1+r-\widehat{s})\left(\left|\mathscr{D}_{3}\right|^{2}+\left|\mathscr{C}_{3}\right|^{2}\right) \\
& +64 m_{\ell}^{2} m_{\mathscr{R}_{Q}}^{3}(1-r-\widehat{s}) \operatorname{Re}\left[\mathscr{D}_{1}^{*} \mathscr{E}_{3}+\mathscr{D}_{3} \mathscr{E}_{1}^{*}\right] \\
& +64 m_{\mathscr{B}_{Q}}^{2} \sqrt{r}\left(6 m_{\ell}^{2}-m_{\mathscr{B}_{Q}}^{2} \widehat{s}\right) \operatorname{Re}\left[\mathscr{D}_{1}^{*} \mathscr{E}_{1}\right] \\
& +64 m_{\ell}^{2} m_{\mathscr{B}_{Q}}^{3} \sqrt{r}\left\{2 m_{\mathscr{B}_{Q}} \widehat{s} \operatorname{Re}\left[\mathscr{D}_{3}^{*} \mathscr{C}_{3}\right]+(1-r+\widehat{s})\right. \\
& \left.+\operatorname{Re}\left[\mathscr{D}_{1}^{*} \mathscr{D}_{3}+\mathscr{E}_{1}^{*} \mathscr{C}_{3}\right]\right\}+32 m_{\mathscr{B}_{Q}}^{2}\left(2 m_{\ell}^{2}+m_{\mathscr{B}_{Q}}^{2} \widehat{s}\right) \\
& +\left\{(1-r+\widehat{s}) m_{\mathscr{B}_{Q}} \sqrt{r} \operatorname{Re}\left[\mathscr{A}_{1}^{*} \mathscr{A}_{2}+\mathscr{B}_{1}^{*} \mathscr{B}_{2}\right]\right. \\
& -m_{\mathscr{B}_{Q}}(1-r-\widehat{s}) \operatorname{Re}\left[\mathscr{A}_{1}^{*} \mathscr{B}_{2}+\mathscr{A}_{2}^{*} \mathscr{B}_{1}\right] \\
& \left.+2 \sqrt{r}\left(\operatorname{Re}\left[\mathscr{A}_{1}^{*} \mathscr{B}_{1}\right]+m_{\mathscr{B}_{Q}}^{2} \widehat{s e}\left[\mathscr{A}_{2}^{*} \mathscr{B}_{2}\right]\right)\right\} \\
& +8 m_{\mathscr{B}_{Q}}^{2}\left\{4 m_{\ell}^{2}(1+r-\widehat{s})+m_{\mathscr{B}_{Q}}^{2}\left[(1-r)^{2}-\widehat{s}^{2}\right]\right\}
\end{aligned}
$$




$$
\begin{aligned}
& \cdot\left(\left|\mathscr{A}_{1}\right|^{2}+\left|\mathscr{B}_{1}\right|^{2}\right) \\
& +8 m_{\mathscr{B}_{Q}}^{4}\left\{4 m_{\ell}^{2}[\lambda+(1+r-\widehat{s}) \widehat{s}]\right. \\
& \left.+m_{\mathscr{B}_{Q}}^{2} \widehat{s}\left[(1-r)^{2}-\widehat{s}^{2}\right]\right\}\left(\left|\mathscr{A}_{2}\right|^{2}+\left|\mathscr{B}_{2}\right|^{2}\right) \\
& -8 m_{\mathscr{B}_{Q}}^{2}\left\{4 m_{\ell}^{2}(1+r-\widehat{s})-m_{\mathscr{B}_{Q}}^{2}\left[(1-r)^{2}-\widehat{s}^{2}\right]\right\} \\
& \cdot\left(\left|\mathscr{D}_{1}\right|^{2}+\left|\mathscr{E}_{1}\right|^{2}\right) \\
& +8 m_{\mathscr{B}_{Q}}^{5} \widehat{s} v^{2}\left\{-8 m_{\mathscr{B}_{Q}} \hat{s} \sqrt{r} \operatorname{Re}\left[\mathscr{D}_{2}^{*} \mathscr{E}_{2}\right]\right. \\
& +4(1-r+\widehat{s}) \sqrt{r} \operatorname{Re}\left[\mathscr{D}_{1}^{*} \mathscr{D}_{2}+\mathscr{E}_{1}^{*} \mathscr{E}_{2}\right] \\
& -4(1-r-\widehat{s}) \operatorname{Re}\left[\mathscr{D}_{1}^{*} \mathscr{C}_{2}+\mathscr{D}_{2}^{*} \mathscr{E}_{1}\right] \\
& \left.+m_{\mathscr{B}_{Q}}\left[(1-r)^{2}-\hat{s}^{2}\right]\left(\left|\mathscr{D}_{2}\right|^{2}+\left|\mathscr{E}_{2}\right|^{2}\right)\right\}, \\
& \mathscr{T}_{1}^{\text {tot }}(\widehat{s})=-16 m_{\mathscr{B}_{Q}}^{4} \widehat{s} 1 v \sqrt{\lambda}\left\{2 \operatorname{Re}\left(\mathscr{A}_{1}^{*} \mathscr{D}_{1}\right)\right. \\
& -2 \operatorname{Re}\left(\mathscr{B}_{1}^{*} \mathscr{E}_{1}\right) \\
& \left.+2 m_{\mathscr{B}_{Q}} \operatorname{Re}\left(\mathscr{B}_{1}^{*} \mathscr{D}_{2}-\mathscr{B}_{2}^{*} \mathscr{D}_{1}+\mathscr{A}_{2}^{*} \mathscr{E}_{1}-\mathscr{A}_{1}^{*} \mathscr{E}_{2}\right)\right\} \\
& +32 m_{\mathscr{B}_{Q}}^{5} \hat{s} v \sqrt{\lambda}\left\{m_{\mathscr{B}_{Q}}(1-r) \operatorname{Re}\left(\mathscr{A}_{2}^{*} \mathscr{D}_{2}-\mathscr{B}_{2}^{*} \mathscr{C}_{2}\right)\right. \\
& \left.+\sqrt{r} \operatorname{Re}\left(\mathscr{A}_{2}^{*} \mathscr{D}_{1}+\mathscr{A}_{1}^{*} \mathscr{D}_{2}-\mathscr{B}_{2}^{*} \mathscr{E}_{1}-\mathscr{B}_{1}^{*} \mathscr{E}_{2}\right)\right\}, \\
& \mathscr{T}_{2}^{\text {tot }}(\widehat{s})=-8 m_{\mathscr{B}_{Q}}^{4} v^{2} \lambda\left(\left|\mathscr{A}_{1}\right|^{2}+\left|\mathscr{B}_{1}\right|^{2}+\left|\mathscr{D}_{1}\right|^{2}\right. \\
& \left.+\left|\mathscr{E}_{1}\right|^{2}\right)+8 m_{\mathscr{B}_{Q}}^{6} \widehat{s} v^{2} \lambda\left(\left|\mathscr{A}_{2}\right|^{2}+\left|\mathscr{B}_{2}\right|^{2}+\left|\mathscr{D}_{2}\right|^{2}\right. \\
& \left.+\left|\mathscr{E}_{2}\right|^{2}\right) \text {. }
\end{aligned}
$$

\section{Conflicts of Interest}

The authors declare that there are no conflicts of interest regarding the publication of this paper.

\section{Acknowledgments}

K. Azizi acknowledges Doğuş University for the financial support through Grant BAP 2015-16-D1-B04.

\section{References}

[1] CDF Collaboration, "Observation of the Baryonic FlavorChanging Neutral Current Decay $\Lambda_{b} \rightarrow \Lambda \mu^{+} \mu^{-}$," Physical Review Letters, vol. 107, article 201802, 2011.

[2] The LHCb Collaboration, "Differential branching fraction and angular analysis of $\Lambda_{b}^{0} \rightarrow \Lambda \mu^{+} \mu^{-}$decays," JHEP, vol. 1506, no. 115, 2015.

[3] T. M. Aliev, K. Azizi, and M. Savci, "Analysis of the $\Lambda_{b} \rightarrow \Lambda l^{+} l^{-}$ decay in QCD," Physical Review D-Particles, Fields, Gravitation and Cosmology, vol. 81, article 056006, no. 5, 2010.

[4] K. Azizi, M. Bayar, A. Ozpineci, Y. Sarac, and H. Sundu, "Semileptonic transition of $\Sigma_{b}$ to $\Sigma$ in light cone QCD sum rules," Physical Review D-Particles, Fields, Gravitation and Cosmology, vol. 85, article 016002, no. 1, 2012.

[5] K. Azizi, Y. Sarac, and H. Sundu, "Light cone QCD sum rules study of the semileptonic heavy $\Xi \mathrm{Q}$ and $\Xi^{\prime} \mathrm{Q}$ transitions to $\Xi$ and $\Sigma$ baryons," European Physical Journal A, vol. 48, no. 1, article no. 2, pp. 1-13, 2012.

[6] K. Azizi, S. Kartal, N. Katirci, A. T. Olgun, and Z. Tavukoglu, "Constraint on compactification scale via recently observed baryonic $\Lambda_{b} \rightarrow \Lambda \ell^{+} \ell^{-}$channel and analysis of the $\Sigma_{b} \rightarrow \Sigma \ell^{+} \ell^{-}$ transition in SM and UED scenario," Journal of High Energy Physics, vol. 2012, no. 5, article no. 024, 2012.

[7] K. Azizi, S. Kartal, A. T. Olgun, and Z. Tavukoglu, "Analysis of the semileptonic $\Lambda_{b} \rightarrow \Lambda \ell^{+} \ell^{-}$transition in the topcolorassisted technicolor model," Physical Review D, vol. 88, article 075007, no. 7, 2013.

[8] B. Dumont, K. Nishiwaki, and R. Watanabe, "LHC constraints and prospects for S1 scalar leptoquark explaining the $\bar{B} \rightarrow$ $D^{(*)} \tau \bar{\nu}$ anomaly," Physical Review D - Particles, Fields, Gravitation and Cosmology, vol. 94, no. 3, Article ID 034001, 2016.

[9] K. A. Olive, "Particle Data Group," Chinese Physics C, vol. 38, article 090001, 2014.

[10] I. Doršner, S. Fajfer, A. Greljo, J. Kamenik, and N. Košnik, "Physics of leptoquarks in precision experiments and at particle colliders," Physics Reports, vol. 641, pp. 1-68, 2016.

[11] S. Davidson, D. Bailey, and B. A. Campbell, "Model independent constraints on leptoquarks from rare processes," Zeitschrift für Physik C Particles and Fields, vol. 61, no. 4, pp. 613-643, 1994.

[12] J. L. Hewett and T. G. Rizzo, "Much ado about leptoquarks: A comprehensive analysis," Physical Review D, vol. 56, no. 9, pp. 5709-5724, 1997.

[13] O. Shanker, "Flavour violation, scalar particles and leptoquarks," Nuclear Physics, Section B, vol. 206, no. 2, pp. 253-272, 1982.

[14] S. Fajfer and N. Košnik, "Leptoquarks in FCNC decays," Physical Review D, vol. 79, article 017502, no. 1, 2009.

[15] J. P. Saha, B. Misra, and A. Kundu, "Constraining scalar leptoquarks from the K and B Sectors," Physical Review D, vol. 81, article 095011, no. 9, 2010.

[16] S. Davidson and P. Verdier, "Leptoquarks decaying to a top quark and a charged lepton at hadron colliders," Physical Review $D$, vol. 83, article 115016, 2011.

[17] N. Košnik, "Model independent constraints on leptoquarks from $b \rightarrow s \ell^{+} \ell^{-}$processes," Physical Review $D$, vol. 86, article 055004, no. 5, 10 pages, 2012.

[18] J. M. Arnold, B. Fornal, and M. B. Wise, "Phenomenology of scalar leptoquarks," Physical Review D, vol. 88, article 035009, no. 3, 2013.

[19] Y. Sakaki, R. Watanabe, M. Tanaka, and A. Tayduganov, “Testing leptoquark models in $\bar{B} \rightarrow D^{(*)} \tau \bar{\nu}$," Physical Review D, vol. 88, article 094012, no. 9, 2013.

[20] R. Mohanta, "Effect of scalar leptoquarks on the rare decays of Bs meson," Physical Review D, vol. 89, article 014020, no. 1, 2014.

[21] B. Allanach, A. Alves, F. S. Queiroz, K. Sinha, and A. Strumia, "Interpreting the CMS $\ell^{+} \ell^{-} j j E_{\mathrm{T}}$ excess with a leptoquark model," Physical Review D, vol. 92, article 055023, no. 5, 2015.

[22] S. Sahoo and R. Mohanta, "Scalar leptoquarks and the rare B meson decays," Physical Review D - Particles, Fields, Gravitation and Cosmology, vol. 91, article 094019, no. 9, Article ID 094019, 2015. 
[23] S. Sahoo and R. Mohanta, "Study of the rare semileptonic decays $B_{d}^{0} \rightarrow K^{*} l^{+} l^{-}$in scalar leptoquark model," Physical Review D, vol. 93, article 034018, no. 3, 20 pages, 2016.

[24] S. Sahoo and R. Mohanta, "Leptoquark effects on and $b \rightarrow$ svvfi and $B \rightarrow K I^{+} I^{-}$decay processes," New Journal of Physics, vol. 18, article 013032, no. 1, 2016.

[25] S. Sahoo and R. Mohanta, "Lepton flavor violating B meson decays via a scalar leptoquark," Physical Review D - Particles, Fields, Gravitation and Cosmology, vol. 93, no. 11, Article ID 114001, 2016.

[26] G. Kumar, "Constraints on a scalar leptoquark from the kaon sector," Physical Review D - Particles, Fields, Gravitation and Cosmology, vol. 94, article 014022, no. 1, 2016.

[27] I. Doršner, S. Fajfer, and N. Košnik, "Can scalar leptoquarks explain the fDs puzzle?" Physical Review D, vol. 682, no. 67, 2009.

[28] I. Doršner, J. Drobnak, S. Fajfer, J. F. Kamenik, and N. Košnik, "Limits on scalar leptoquark interactions and consequences for GUTs," Journal of High Energy Physics, vol. 2011, no. 11, article no. 002, 2011.

[29] D. Bečirević, N. Košnik, O. Sumensari, and R. Zukanovich Funchal, "Palatable leptoquark scenarios for lepton flavor violation in exclusive $b \rightarrow \mathrm{s} \ell_{1} \ell_{2}$ modes," Journal of High Energy Physics, vol. 2016, no. 11, article no. 35, 2016.

[30] D. Bečirević, S. Fajfer, N. Košnik, and O. Sumensari, "Leptoquark model to explain the B-physics anomalies, RK and RD," Physical Review D, vol. 94, article 115021, no. 11, 2016.

[31] W. Detmold and S. Meinel, " $\Lambda_{b} \rightarrow \Lambda \ell^{+} \ell^{-}$form factors, differential branching fraction, and angular observables from lattice QCD with relativistic $b$ quarks," Physical Review D, vol. 93, article 074501, no. 7, 2016.

[32] G. Buchalla, A. J. Buras, and M. E. Lautenbacher, "Weak decays beyond leading logarithms," Reviews of Modern Physics, vol. 68, no. 4, pp. 1125-1244, 1996.

[33] W. Altmannshofer, P. Ball, A. Bharucha, A. J. Buras, D. M. Straub, and M. Wick, "Symmetries and asymmetries of $B \rightarrow$ $K^{*} \mu^{+} \mu^{-}$decays in the standard model and beyond," Journal of High Energy Physics, vol. 901, no. 019, 2009.

[34] S. Sahoo and R. Mohanta, "Effects of scalar leptoquark on semileptonic $\Lambda$ b decays," New Journal of Physics, vol. 18, article 093051, no. 9, 2016.

[35] T. Feldmann and M. W. Y. Yip, "Form Factors for $\Lambda b \rightarrow \Lambda$ Transitions in SCET," Physical Review D, vol. 86, 2012, article 079901.

[36] P. Ber, T. Feldmann, and D. van Dyk, "Angular analysis of the decay $\Lambda_{b} \rightarrow \Lambda(\rightarrow N \pi) \ell^{+} \ell^{-}$," Journal of High Energy Physics, vol. 1501, no. 155, 2015.

[37] Y. M. Wang and Y. L. Shen, "Perturbative corrections to $\Lambda_{b} \rightarrow \Lambda$ Form Factors from QCD light-cone sum rules," JHEP, vol. 1602, no. 179, 2016.

[38] S. Descotes-Genon, J. Matias, and J. Virto, "Understanding the $B \rightarrow K^{*} \mu^{+} \mu^{-}$anomaly," Physical Review D, vol. 88, article 074002, no. 7, 2013.

[39] T. Hurth, F. Mahmoudi, and S. Neshatpour, "Global fits to $b \rightarrow$ sle data and signs for lepton non-universality," Journal of High Energy Physics, vol. 2014, no. 12, article 53, 2014.

[40] F. Beaujean, C. Bobeth, and S. Jahn, "Constraints on tensor and scalar couplings from $B \rightarrow K \mu \mu$ and $B_{s} \rightarrow \mu \mu$," The European Physical Journal C, vol. 75, article 456, no. 9, 2015.

[41] D. Du, A. X. EI-Khadra, S. Gottlieb et al., "Phenomenology of semileptonic $B$-meson decays with form factors from lattice QCD," Physical Review D, vol. 93, no. 3, Article ID 034005, 2016.
[42] S. Descotes-Genon, L. Hofer, J. Matias, and J. Virto, "Global analysis of $b \rightarrow \mathrm{s} \ell \ell$ anomalies," Journal of High Energy Physics, vol. 1606, article 092, no. 6, 2016.

[43] T. Hurth, F. Mahmoudi, and S. Neshatpour, "On the anomalies in the latest LHCb data," Nuclear Physics B, vol. 909, pp. 737-777, 2016.

[44] S. Meinel and D. Van Dyk, "Using $\Lambda_{b} \rightarrow \Lambda \mu^{+} \mu^{-}$data within a Bayesian analysis of $|\Delta B|=|\Delta S=1|$ decays," Physical Review D - Particles, Fields, Gravitation and Cosmology, vol. 94, article 013007, no. 1, 2016.

[45] S. Wang and Y. Yang, "Analysis of $\Lambda_{b} \rightarrow \Lambda \mu^{+} \mu^{-}$decay in scalar leptoquark model," Advances in High Energy Physics, vol. 2016, Article ID 5796131, 7 pages, 2016.

[46] A. J. Buras, M. Misiak, M. Münz, and S. Pokorski, “Theoretical uncertainties and phenomenological aspects of $B \rightarrow X(s)$ gamma decay," Nuclear Physics, Section B, vol. 424, no. 2, pp. 374-398, 1994.

[47] M. Misiak, "The $b \rightarrow s e^{+} e^{-}$and $b \rightarrow s \gamma$ decays with next-toleading logarithmic QCD-corrections," Nuclear Physics, Section $B$, vol. 393, no. 1-2, pp. 23-45, 1993.

[48] A. J. Buras and M. Münz, "Effective Hamiltonian for $B \rightarrow$ $X(s) e^{+} e^{-}$beyond leading logarithms in the NDR and $\mathrm{HV}$ schemes," Physical Review D, vol. 52, no. 186, 1995.

[49] A. J. Buras, "Weak Hamiltonian, CP violation and rare decays, "to appear in" probing the standard model of particle interactions," in Elsevier Science B.V, F. David and R. Gupta, Eds. 

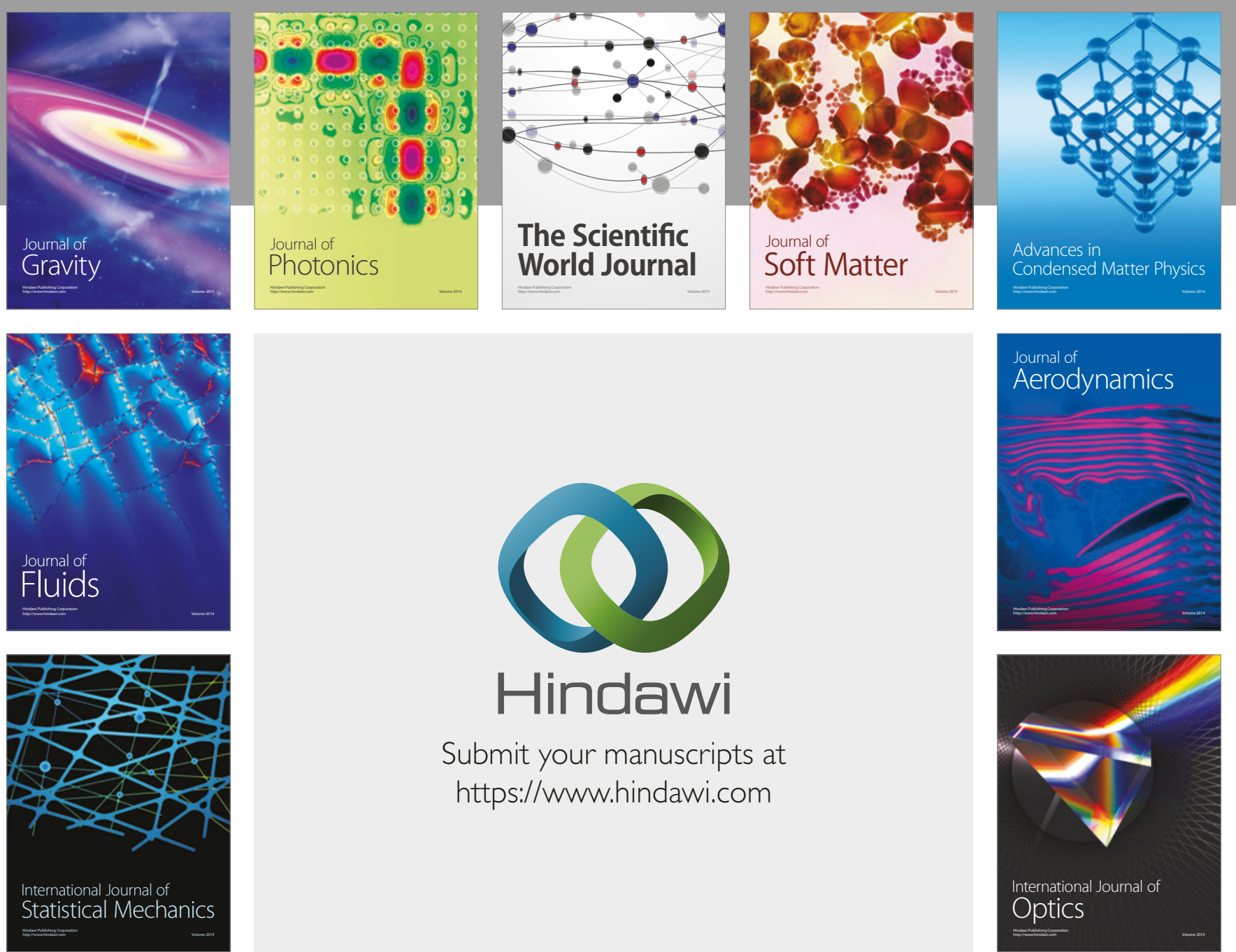

Submit your manuscripts at

https://www.hindawi.com
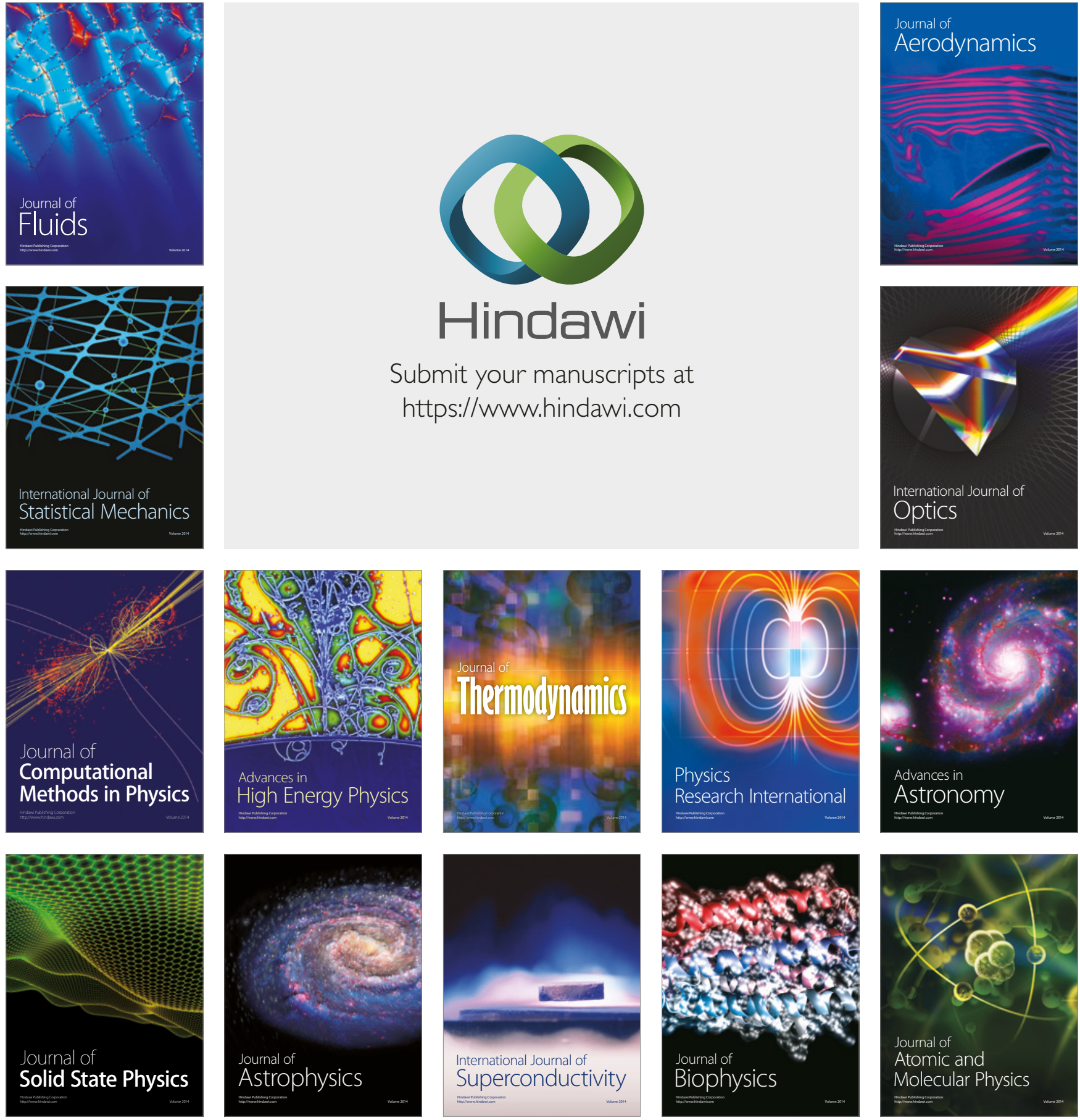